\title{
Iron Quantum Dots Electro-Assembling on Vulcan XC-72R: Hydrogen Peroxide Generation for Space Applications
}

\author{
Armando Peña-Duarte ${ }^{1 *}$, Santosh H. Vijapur ${ }^{2}$, Timothy D. Hall ${ }^{2}$, Kathleen \\ L. Hayes ${ }^{3}$, Eduardo Larios-Rodríguez ${ }^{4}$, Joselyn Del Pilar-Albaladejo ${ }^{5}$, \\ Mitk'El B. Santiago ${ }^{6}$, Stephen Snyder ${ }^{2}$, Jennings Taylor ${ }^{2}$, and Carlos R. \\ Cabrera $^{5 *}$ \\ ${ }^{1}$ Department of Physics, University of Puerto Rico, San Juan, PR 00926, United States. \\ ${ }^{2}$ Faraday Technology Inc., Englewood, OH 45315, United States. \\ ${ }^{3}$ Department of Chemistry and Biochemistry, University of Notre Dame, Notre Dame, IN 46556, \\ United States. \\ ${ }^{4}$ Department of Chemical Engineering, Sonora University, Hermosillo, México 83000. \\ ${ }^{5}$ Department of Chemistry, University of Puerto Rico, San Juan, PR 00926, United States. \\ ${ }^{6}$ Department of Chemistry, Universidad Ana G. Méndez, Cupey Campus, San Juan, PR, 00926, \\ United States. \\ *Corresponding Authors: armando.pena@upr.edu; carlos.cabrera2@upr.edu
}




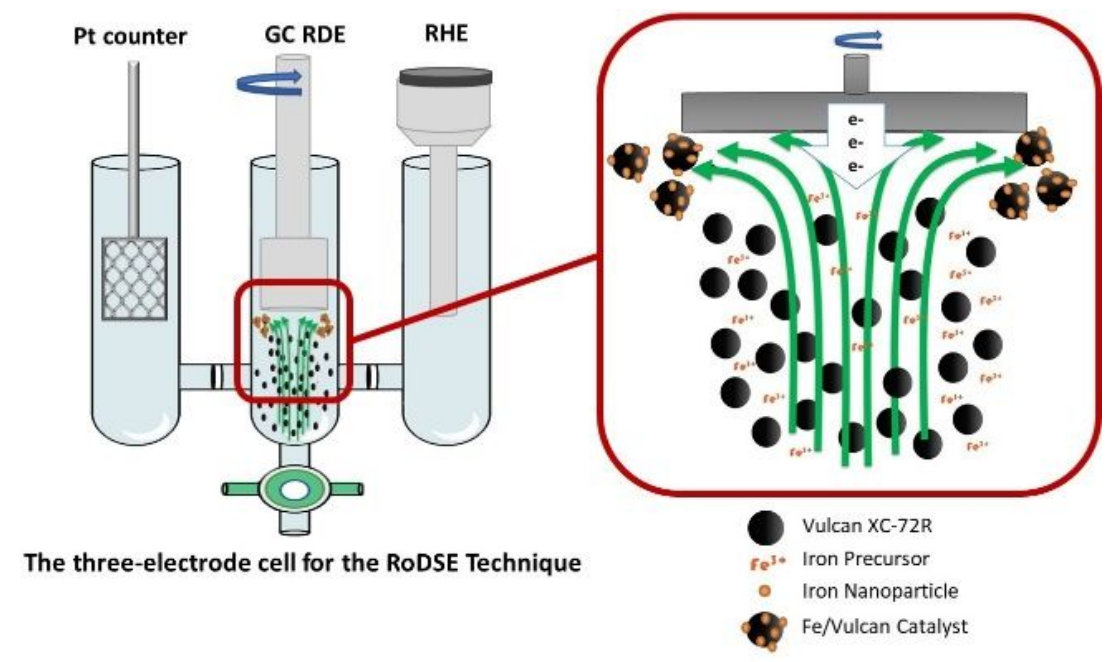

Figure S1. Schematic of the RoDSE Fe/Vulcan XC-72R quantum dots electrochemical synthesis setup in $0.1 \mathrm{M} \mathrm{KOH}$. The three-compartment electrode cell diagram corresponds from right to left to a reversible hydrogen reference electrode, glassy carbon rotating disk working electrode, and Pt auxiliary electrode.

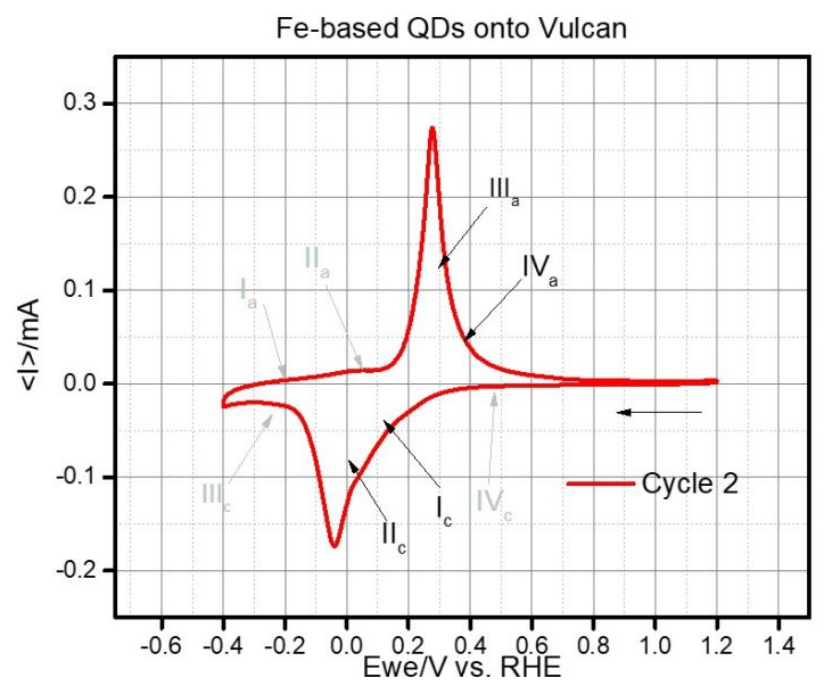

Figure S2. Second cycle of the Fe/Vulcan XC-72R cyclic voltammetry analysis. It was done in a nitrogen-purged $0.1 \mathrm{M} \mathrm{KOH}$ solution. Catalyst surface modifications are evidenced through modifications of the cathodic and anodic peaks in comparison with the first cycle, presented in 

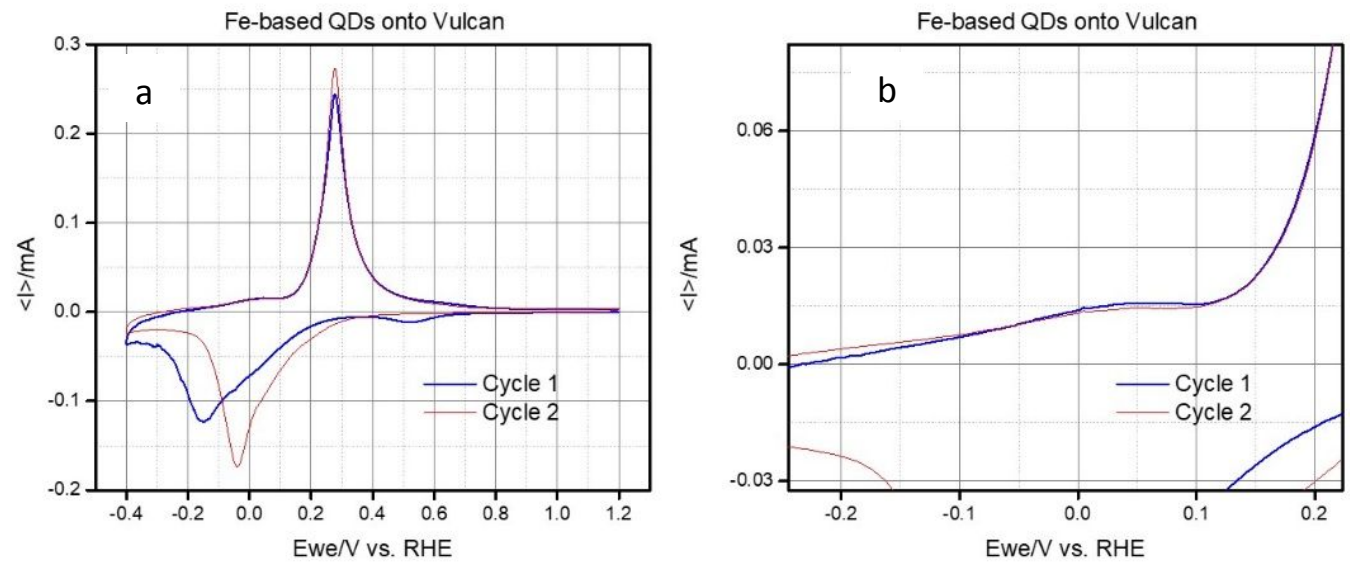

Figure S3. (a) Cyclic voltammetry profile vs. RHE, at a scan rate of $10 \mathrm{mV} / \mathrm{s}$ and at room temperature, of the Fe/Vulcan XC-72R. The deviation of the first cycle, from the successive second cycle, is observed in both reduction and oxidation peaks. The potential is swept in the cathodic direction starting at $0.5 \mathrm{~V}$ and ending at $-0.9 \mathrm{~V}$. (b) A enlarged view of the IIa oxidation peak at the potential window -200 to $200 \mathrm{mV}$ is shown.

Table S1. Induced Coupled Plasma-Optical Emission Spectroscopy (ICP-OES) and metal loading: The quantitative determination of the total iron electrodeposited and its percentage ratio loading efficiency by the RoDSE technique were performed through Induced Coupled Plasma-Optical Emission Spectroscopy (ICP-OES).

Spectrometer Perkin Elmer Optima 8000

$$
\begin{aligned}
& \%_{\text {loading }}=(25.00 \pm 0.03) \% \\
& \%_{\text {FPe }}=(15.54 \pm 0.03) \%
\end{aligned}
$$

Electrodeposition Efficiency $=62.16 \%$ 

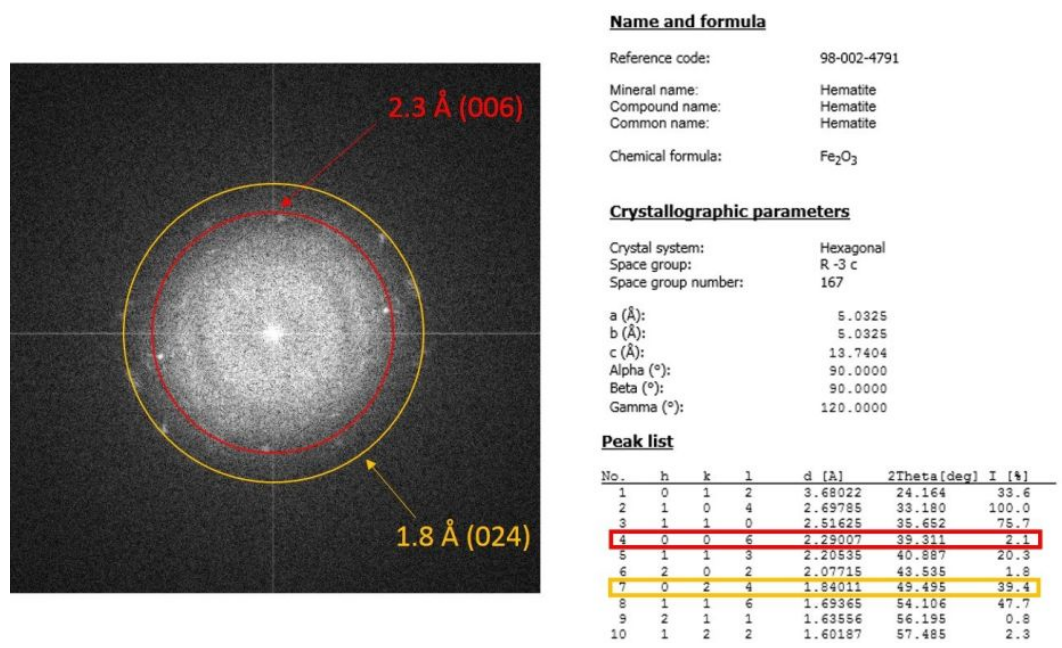

Figure S4. The TEM electron diffraction pattern of the Fe/Vulcan catalyst electrodeposited by the RoDSE technique. The diffraction pattern analysis confirms ironoxide based species.

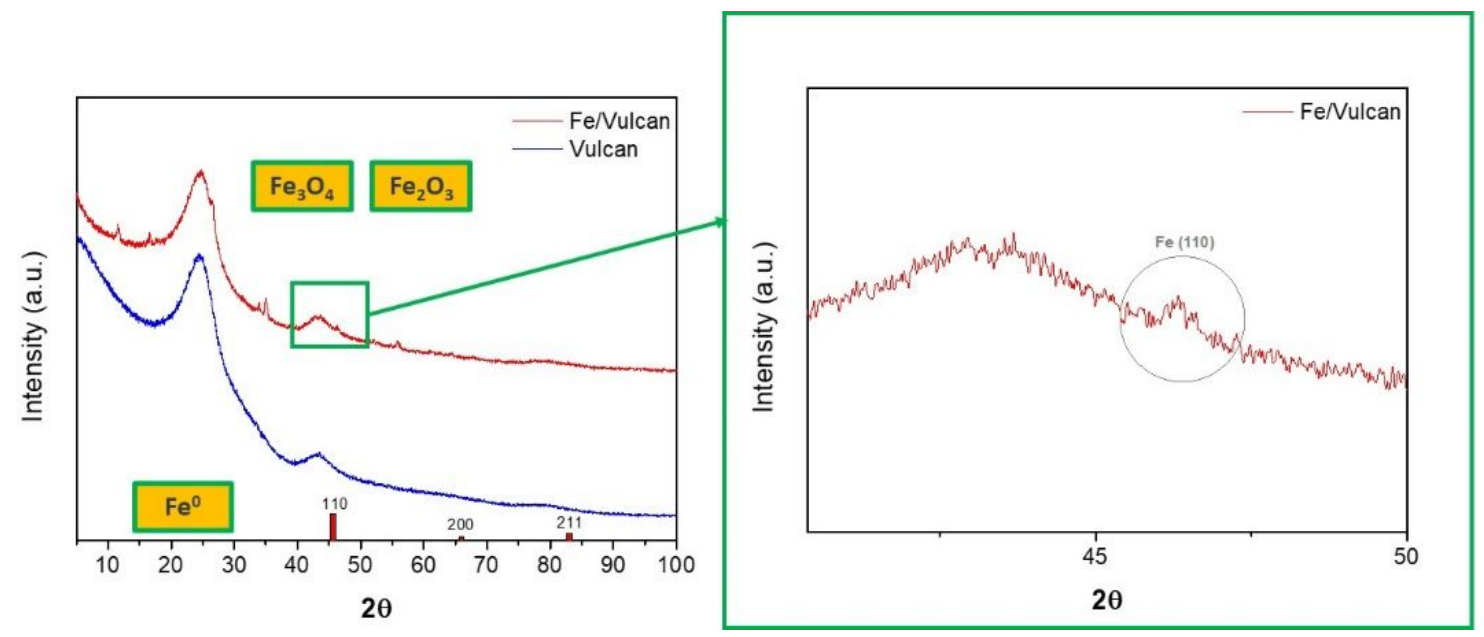

Figure S5. X-ray diffraction (XRD) measurements were performed on fresh iron-based catalyst synthesized by the RoDSE Technique. XRD analysis evidences the successful deposition of iron on the carbon support. The metallic peak (110) in the characteristic $2 \theta$ about $46^{\circ}$ support the presence of the zero valent iron specie in a fresh catalytic sample. The broadness and ill-definition of diffractogram peaks are characteristic very small nanostructures. Oxidation of iron is evidenced by the presence of other peaks associated with pattern of iron oxidizes species. 

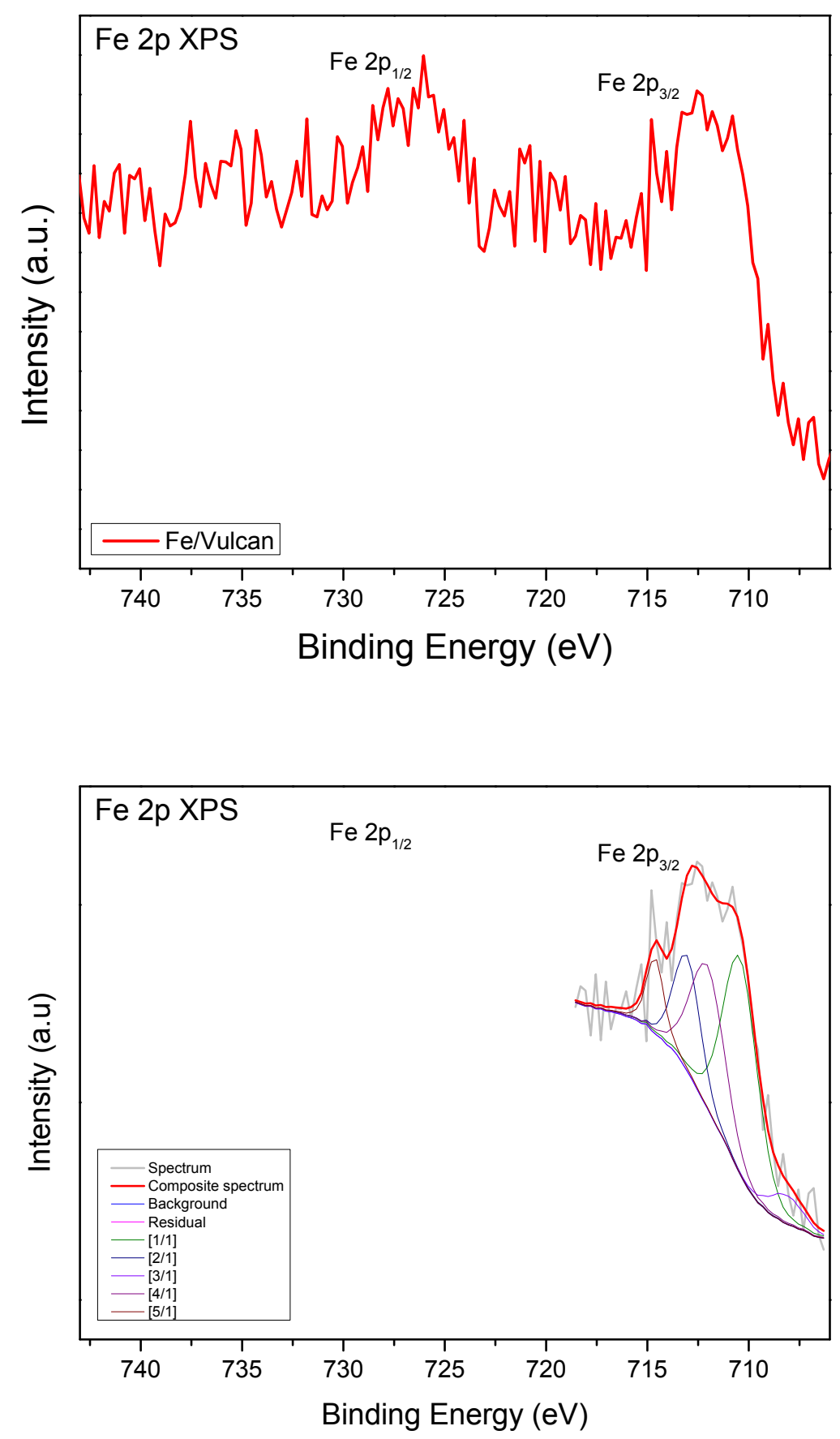

Figure S6. X-ray photoelectron spectroscopy(XPS) Fe $2 p$ binding energy region scan with the Fe $2 p_{1 / 2}$ and $\mathrm{Fe} 2 \mathrm{p}_{3 / 2}$ duplet (top), and the Voigt deconvolution analysis of the Fe $2 p_{3 / 2}$ binding energy region (bottom) for the iron-based QDs onto Vulcan XC-72R electrodeposited by the RoDSE technique. 
Table S2. XPS Peaks Assignments for the C 1s Components

\begin{tabular}{cc}
\hline C 1s Components & Binding Energy (eV) \\
\hline Graphite $\left(\mathrm{sp}^{2}\right)$ & 284.5 \\
$\mathrm{C}-\mathrm{H}\left(\mathrm{sp}^{3}\right)$ & 285.0 \\
$\mathrm{C}-\mathrm{OH} / \mathrm{C}-\mathrm{O}-\mathrm{C}\left(\mathrm{sp}^{3}\right)$ & $286.1-286.3$ \\
$\mathrm{C}=\mathrm{O}$ & $287.6-287.7$ \\
$\mathrm{COOH} / \mathrm{COOR}$ & $288.6-289.1$ \\
$\pi-\pi^{*}$ & $290.5-290.8$ \\
\hline
\end{tabular}

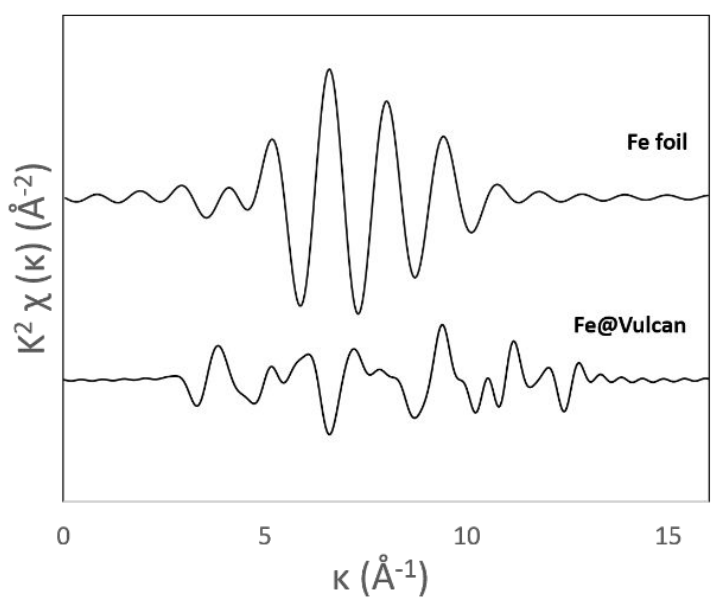

Figure S7. XAFS data at the Fe edge for Fe/Vulcan catalyst and the Fe foil.

Table S3. Quantitative ORR kinetics parameters calculated for the ORR in $0.1 \mathrm{M} \mathrm{KOH}$

\begin{tabular}{cccc}
\hline Catalyst & $\mathbf{E}_{\text {onset }}(\mathbf{V}$ vs RHE $)$ & $\mathbf{E}_{\mathbf{1} / \mathbf{2}}(\mathbf{V}$ vs RHE) & \# of electrons \\
\hline $\mathrm{Fe} /$ Vulcan & 0.566 & 0.467 & 2.6 \\
$\mathrm{Vulcan}$ & 0.642 & 0.573 & 1.6 \\
$\mathrm{Pt}(20 \%) / \mathrm{GC}$ & 0.876 & 0.785 & 4.0 \\
\hline
\end{tabular}



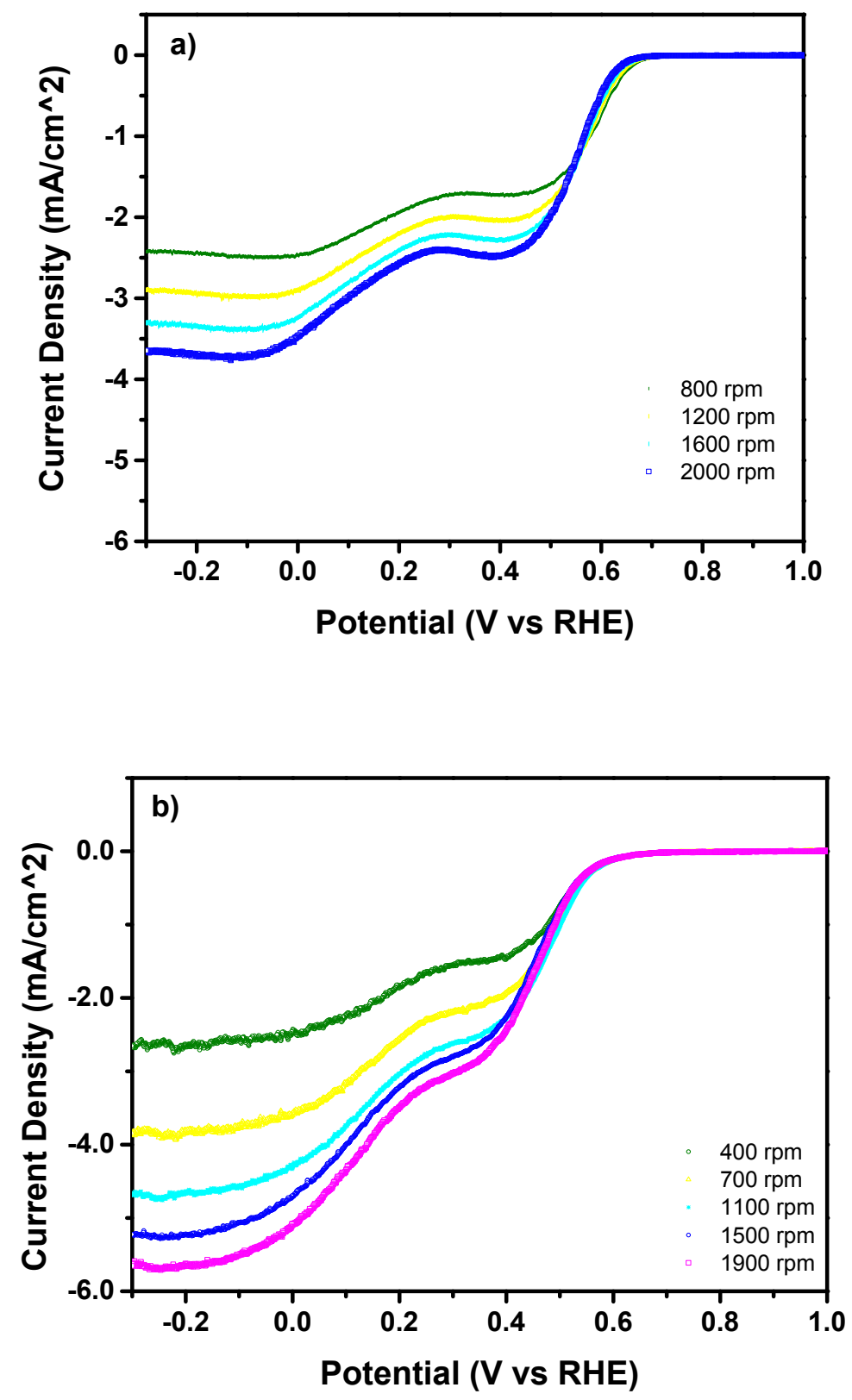

Figure S8. Rotating-disk electrode polarization curves for the Vulcan XC-72R (a) and $\mathrm{Fe} / \mathrm{Vulcan}$ (b) catalyst, electrodeposited by the RoDSE technique. The Koutechy-Levich $(\mathrm{K}-\mathrm{L})$ analysis was done in $\mathrm{O}_{2}$-saturated $0.1 \mathrm{M} \mathrm{KOH}$ solution at a scan ratio of $10 \mathrm{mV} / \mathrm{s}$. at different rotation speeds between 400 and $2400 \mathrm{rpm}$. The linear sweep voltammetry was done form $1.0 \mathrm{~V}$ to $-0.3 \mathrm{~V}$ vs RHE. 

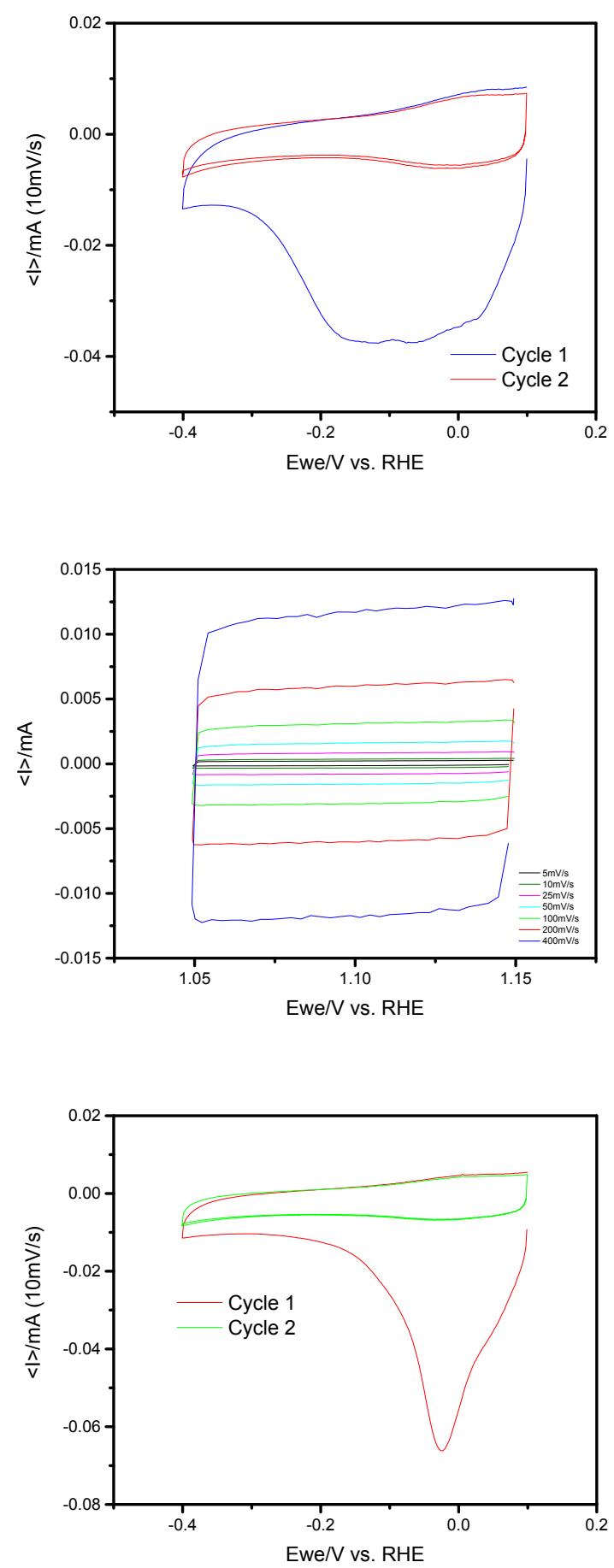

Figure S9. Normalization and tuning-treatment before the RDE and RRDE analyses to assure that all the iron is reduced to $\mathrm{Fe}^{0}$ before to start the ORR kinetics study. The iron reduction peak disappears after the first scanning process, indicating that iron species on the surface reduces to $\mathrm{Fe}^{0}$. 


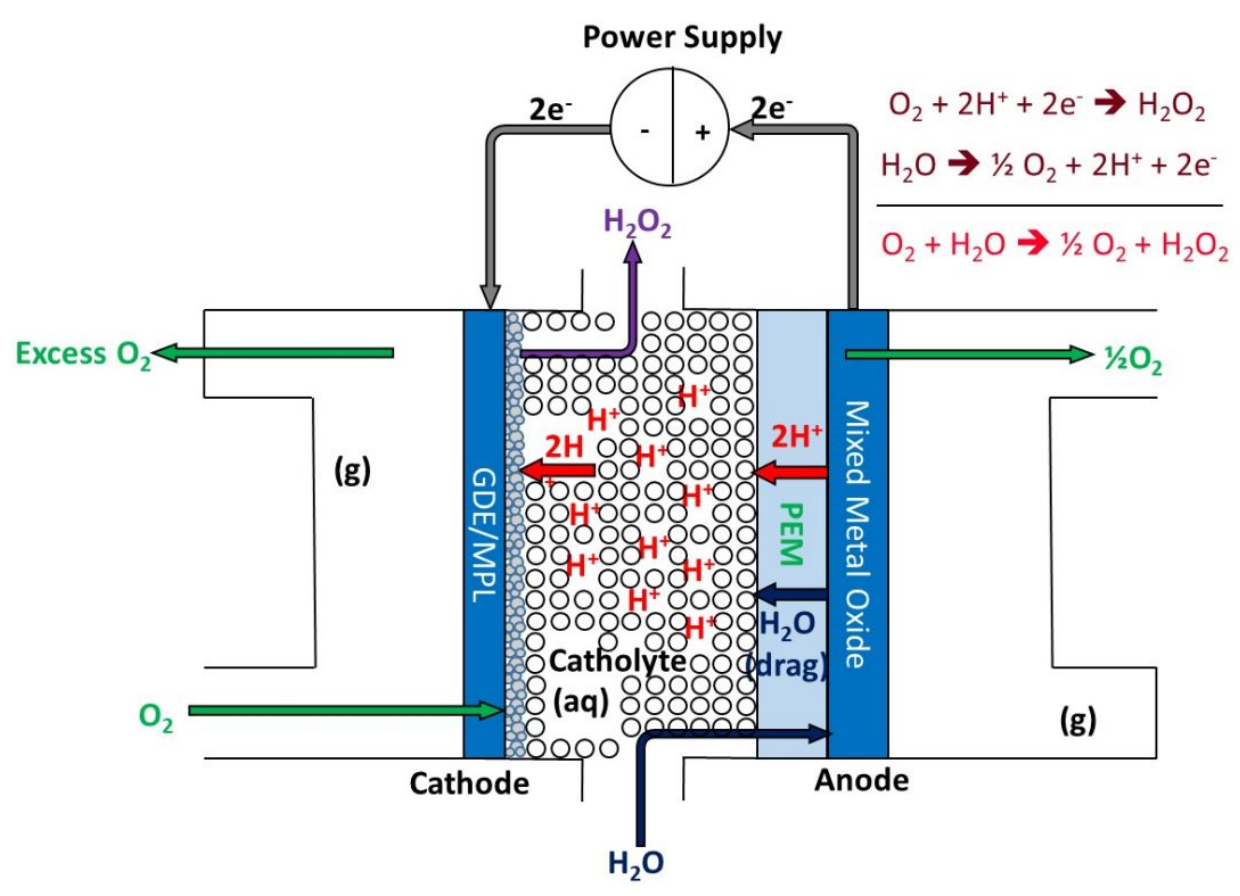

Figure S10. Conceptual schematic of peroxide generation system ${ }^{1,2}$

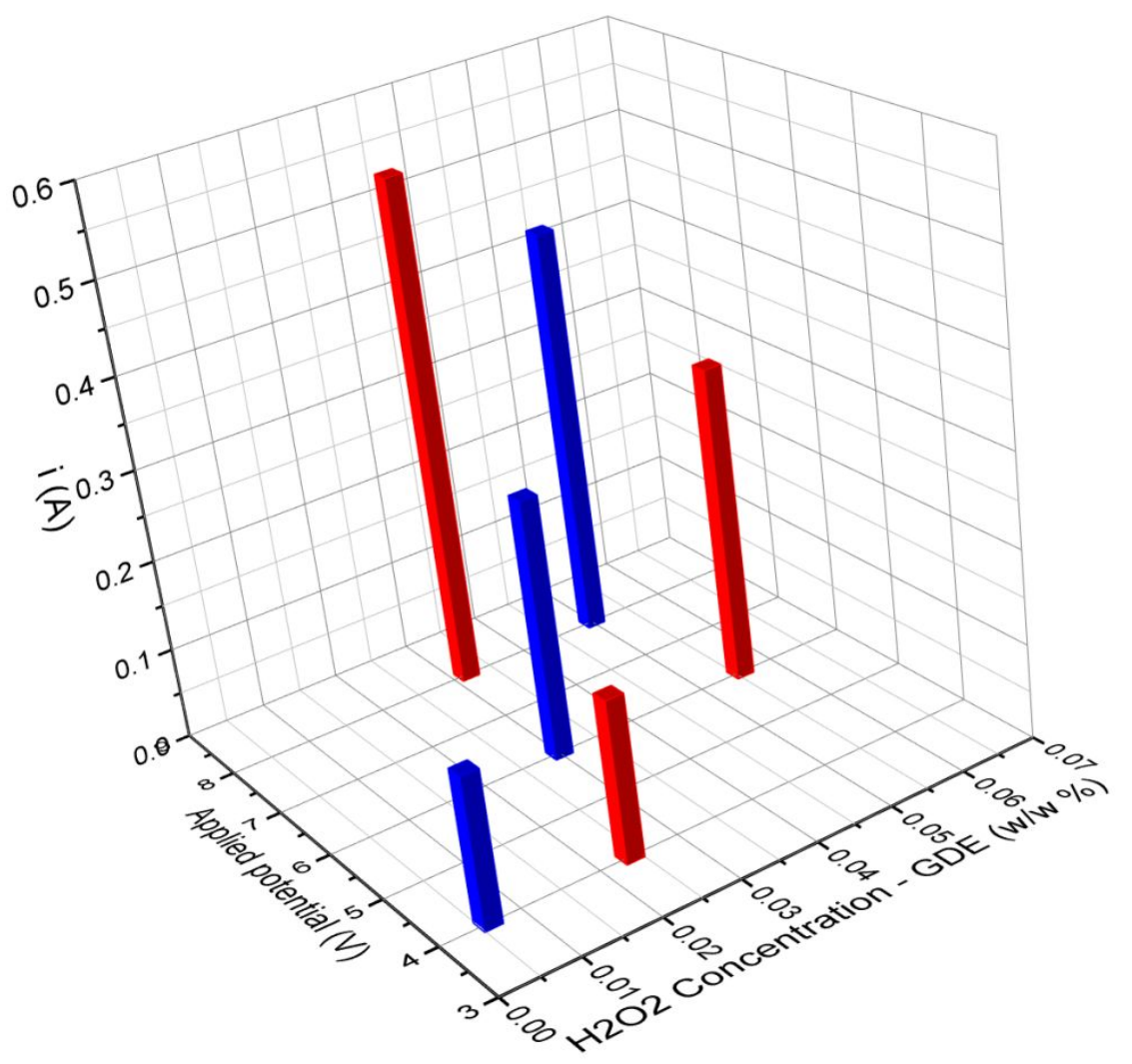

Figure S11. Comparison of hydrogen peroxide production and the output current by $\mathrm{Fe} /$ Vulcan and GDE-MPL at $6 \mathrm{~V}$, using RO water in the peroxide generation unit (PGU) for space applications. Experiments were done at room temperature under terrestrial conditions. 


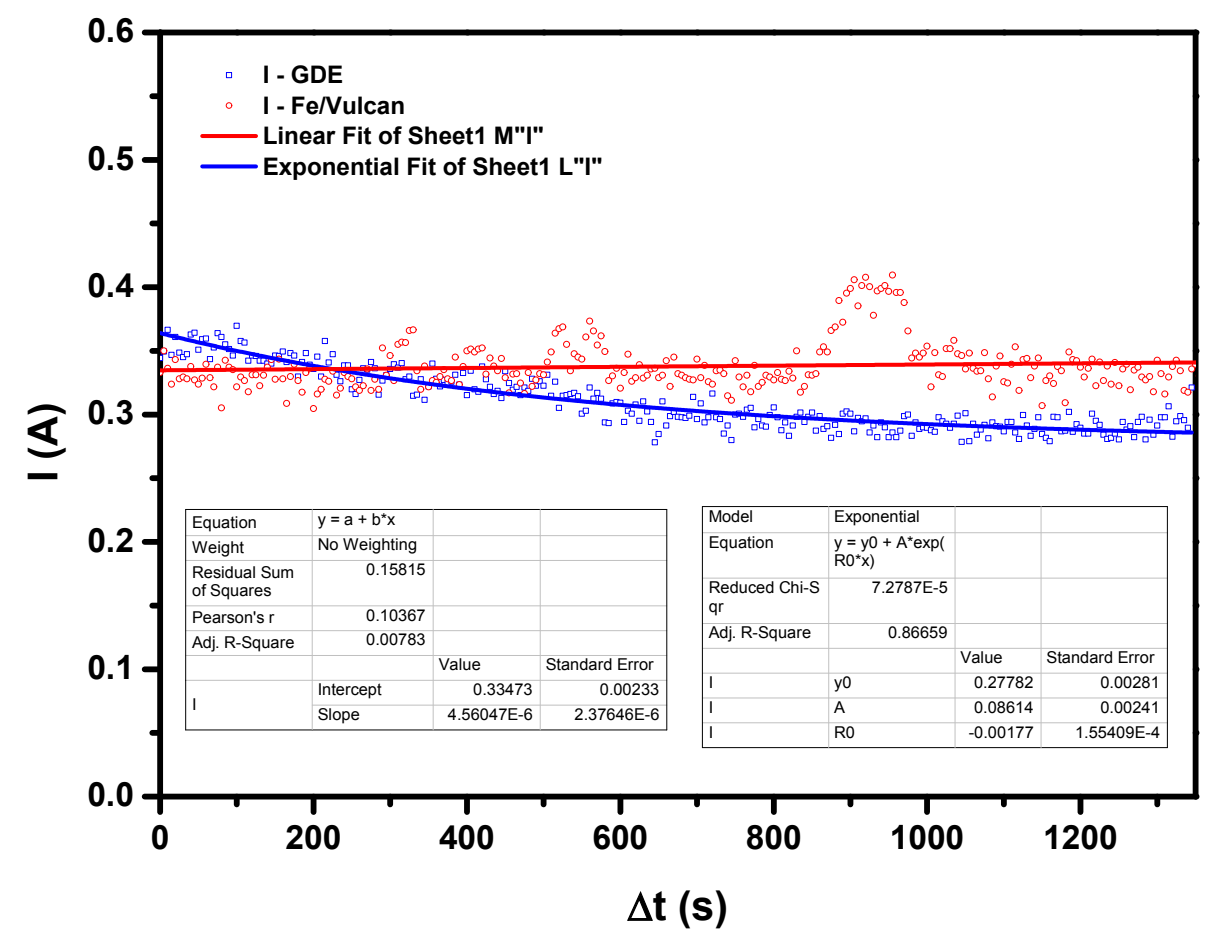

Figure S12. Polarization curve for the in-situ hydrogen peroxide generation on the GDEMPL at $6 \mathrm{~V}$. Blue line shows the output current of the GDE-MPL decreases significantly as time goes by. Its exponential decay behavior indicates a possible decomposition of the carbon in the surface GDE, and a consequently penetration of water in the cathode---after opening the PGU, we observe water presence in the cathodic side. In fact, the GDE trial current decrease exponentially in seconds from $(3120 \mathrm{sec}, 0.315 \mathrm{amps})$ to $(3840 \mathrm{sec}$, $0.281 \mathrm{amps})$, with (time, current) as (x, y) coordinates. Red line shows a comparative behavior of the output currents for the Fe/Vulcan and the GDE-MPL at $6 \mathrm{~V}$.

\section{References:}

1. Vijapur, S. H.; Hall, T. D.; Taylor, E.; Wang, D.; Snyder, S.; Skinn, B.; Peña-Duarte, A.; Cabrera, C. R.; Sweterlitsch, J., In- Situ Resource Utilization for Electrochemical Generation of Hydrogen Peroxide for Disinfection. ICES: International Conference on Environmental Systems 2019, 38, 14 pages.

2. Nelson, G. J.; Vijapur, S. H.; Hall, T. D.; Brown, B. R.; Peña-Duarte, A.; Cabrera, C. R., Electrochemistry for Space Life Support. Electrochemical Society Interface 2020, 29 (1), 47-52. 